\title{
Photoinduced work function modifications and their effect on photoelectron
}

spectroscopy

Daniel Wegkamp, ${ }^{\text {a) }}$ Michael Meyer, Clemens Richter, Martin Wolf, and Julia Stähler Department of Physical Chemistry, Fritz Haber Institute of the Max Planck Society, Faradayweg 4-6, 14195 Berlin, Germany

(Dated: 16 September 2013)

We investigate the effect of a spatially varying work function on photoemission experiments. It is demonstrated that a photoinduced work function change when probed by ultraviolet and two-photon photoemission spectroscopy can have pronounced effects on photoemission spectra. These effects are simulated by a simple model that reproduces the data remarkably well and allows for quantitative interpretation of the modified low energy region of the photoemission spectra. These findings are highly relevant when discussing work function determinations by photoemission spectroscopy and moreover may have substantial impact on the energy level alignment of molecule-metal or -semiconductor interfaces.

a)Electronic mail: wegkamp@fhi-berlin.mpg.de 
The work function $(\Phi)$ of a system is a signature of the surface electronic structure and influences the energy level alignment of frontier molecular orbitals on metal or semiconductor surfaces ${ }^{1}$. This affects, for example, the charge carrier injection in layered systems and devices. For instance, in organic molecule-based electronics, the energy level alignment determines the hole injection barrier from the electrode to the organic layer and thereby the functionality and performance of the device ${ }^{2,3}$. This is because the energy of electronic states that are pinned to the vacuum level (e.g. surface and image potential states, certain molecular levels of weakly bound adsorbates, etc.) are directly connected to the work function $^{4,5}$.

Mechanisms leading to work function changes can be manifold: The charge distribution at the surface strongly influences the (electric) surface dipole and thereby the work function ${ }^{6}$. The specific surface electronic structure itself ${ }^{7}$, charge transfer due to chemisorption ${ }^{8-10}$ and adsorption of dipolar molecules ${ }^{11-13}$ can alter the surface dipole. Also, the irradiation of adsorbed molecules with photons can lead to a change of $\Phi$ by, for instance, photodesorption ${ }^{14}$, photoinduced charge transfer ${ }^{15}$, or other types of photochemistry ${ }^{16,17}$. As light is frequently used for spectroscopic investigations, such photoinduced modifications may affect a large number of studies without being recognized.

Photoemission spectroscopy is frequently used to study the electronic band structure and can also directly probe the work function of a sample. Its spectroscopic signature, the low energy cut-off in the spectra, is furthermore used to determine the resolution of the experimental setup. A variation of the work function - especially when induced by the light source used in the experiment - drastically modifies the energy resolution and the interpretation of the spectroscopic results.

In this Letter, we investigate the influence of photoinduced work function changes on the spectroscopic signatures in ultraviolet photoelectron or single-photon photoemission (UPS or 1PPE) spectroscopy and two-photon photoemission (2PPE) spectroscopy. Besides a broadening and shift of the low energy cut-off, we find that, using a photon energy close to the system's work function distribution, the transition from single- to two-photon photoemission leads to a very distinct spectroscopic signature in the low-energy region of the spectra. This is simulated using a simple model based on a spatially variable work function distribution only. Varying the model parameters allows for a quantitative analysis of the work function distribution. 
The photoemission experiments have been conducted in a ultrahigh vacuum chamber at a base pressure below $1 \times 10^{-10}$ mbar. We used amorphous $\mathrm{D}_{2} \mathrm{O}$ layers of 20 bilayer $(\mathrm{BL})$ thickness on a $\mathrm{Cu}(111)$ single crystal in our investigation, as these samples exhibit a photoinduced permanent work function change of up to $1 \mathrm{eV}$. The $\mathrm{Cu}(111)$ surface was cleaned by Ar-ion sputtering and annealing cycles. The $\mathrm{D}_{2} \mathrm{O}$ layers have been deposited from gas-phase $\mathrm{D}_{2} \mathrm{O}$ (fisher scientific, purity min. $99.95 \%$ ) via a pinhole doser at low sample temperatures $(40 \mathrm{~K})$. The third $(\sim 4.6 \mathrm{eV})$ and fourth $(\sim 6.2 \mathrm{eV})$ harmonic of a Ti:Sa regenerative amplifier system running at $200 \mathrm{kHz}$ repetition rate with a pulse durations of less than $100 \mathrm{fs}$ were used for photoemission. Photoelectrons were detected using a hemispherical electron energy analyzer (SPECS Phoibos 100). In UPS or 1PPE spectroscopy a single photon is absorbed in the sample leading to excitation of an electron above the vacuum level and subsequent photoemission, while in 2PPE a first photon excites an electron into unoccupied bound states and a second photon is needed to lift the electron above the vacuum level which thereby probes the transient excited state population. In both cases the vacuum level (work function) determines the energetic position of the low energy cut-off of the spectrum. Care has been taken to exclude the influence of the analyzer work function by applying a bias voltage of -1.0 to $-1.5 \mathrm{~V}$.

First we investigate the effect of a spatially varying work function on UPS spectra. We imprint such a work function distribution permanently on our $\mathrm{D}_{2} \mathrm{O} / \mathrm{Cu}(111)$ sample by illuminating it with a large diameter $(\mathrm{FWHM} \sim 110 \mu \mathrm{m}) 4.6 \mathrm{eV}$ beam centered at $x=0$. A smaller $($ FWHM $\sim 25 \mu \mathrm{m}$ ) low-intensity $6.2 \mathrm{eV}$ beam is used to spatially scan over the previously illuminated area. The resulting UPS spectra are shown in the false color plot versus sample position $x$ in Fig. 1(a). The FWHM of the beams are indicated by the circles at the top of the figure. The illumination with a large diameter $4.6 \mathrm{eV}$ beam clearly affects the photoemission spectra. In Fig. 1(b) we show single UPS spectra at specific locations (corresponding to the black lines (1-6) in Fig. 1(a)). For the position that is illuminated by the highest intensity (spectrum 1) the position of the low energy cut-off at half maximum (indicated by the red cross) is shifted by $0.5 \mathrm{eV}$ to higher energy in respect to the the cutoff at a sample position that has not been illuminated (spectrum 6). Also the cut-off gets substantially broadened by illumination. The cut-off positions of the spectra (1-5) follow the spatial beam profile of the $4.6 \mathrm{eV}$ laser beam and correspond to the local average work function of the sample. The complete work function profile imprinted onto the sample is 
indicated by the blue line in Fig. 1(a).

The effect of averaging over such a work function distribution is shown at the bottom of Fig. 1(b) for different probe spot sizes centered at $x=0$ (indicated by the curves at the bottom of Fig. 1(a)). The dotted line corresponds to a spot size of $100 \mu \mathrm{m}$ (FWHM) and the resulting edge position is $5.0 \mathrm{eV}$. Only changing the probe spot size to $300 \mu \mathrm{m}$ shifts the apparent cut-off to $4.75 \mathrm{eV}$ while also changing its width. This clearly shows that the value for $\Phi$ itself is strongly affected when sampling over a certain region of a spatially varying work function and cannot simply be extracted via the cut-off position. The immense broadening of the low energy cut-off of the UPS spectra can mask the real resolution for spectral features that are not influenced by the work function distribution.

We now concentrate on the implications of inhomogeneous work function distributions on photoemission spectroscopy when using photon energies similar to typical sample work functions (3-6 eV), as for example used in time-resolved pump-probe experiments using laser-based light sources. Here the photon energies are commonly generated by nonlinear optical processes (second harmonic generation, etc.) which are limited to maximum energies slightly above $6 \mathrm{eV}$ when using a nonlinear optical crystal-based mixing approach ${ }^{18}$. In particular when applying 2PPE spectroscopy, photon energies slightly below the work function are very common, as electrons are intended to be excited into unoccupied states, instead of being emitted by a single photon.

UPS/1PPE has a significantly (approximately 4 orders of magnitude ${ }^{19}$ ) higher crosssection than a two-photon process. Given the finite dynamic range of the photoelectron energy analyzer, two-photon photoemission is only efficiently detected, i.e. without a large single-photon background, if $\mathrm{h} \nu<\Phi<2 \mathrm{~h} \nu$.

Assuming that the work function of the system under investigation depends on the spatial coordinate $\mathrm{x}$ (Fig. 2(a)), then, for the values $\mathrm{x}$ where $\Phi(x)<\mathrm{h} \nu$ the photoemission spectrum will be dominated by 1PPE, whereas if $\Phi(x)>\mathrm{h} \nu$ only $2 \mathrm{PPE}$ can contribute. If $\Phi_{\min }<\mathrm{h} \nu<\Phi_{\max }$ a mixture of both can be observed.

A photoemission spectrum of previously illuminated $\mathrm{D}_{2} \mathrm{O} / \mathrm{Cu}(111)$ (photon energy for photoemission: $4.6 \mathrm{eV}$ ) is shown in Fig. 2(b). The size of the spot that modifies the work function is the same as the one used for photoemission in this case. In contrast to the UPS data there is an additional peak-like feature at the low energy cut-off region of the spectrum. To test whether the non-uniform work function of the sample is responsible for the 
appearance of this feature alone we simulate our data by a simple numerical model.

In the following, we describe this model in detail and discuss the dependence of the work function within the probed area of the sample on the laser intensity. As a start we assume the following formula to describe the dependence of $\Phi(\mathrm{I})$ displayed in Fig. 2(c):

$$
\Phi(I)=\Phi_{0}+\Delta \Phi_{\max }\left(1-e^{-\gamma I}\right) /\left(1-e^{-\gamma}\right) .
$$

The work function $\Phi(I)$ in Equation 1 varies from $\Phi_{0}$ to $\Phi\left(I_{\max }\right)$ depending on the intensity distribution inside the laser spot and the model parameter $\gamma$. In the case of a gaussian beam profile Equation 1 results in a high work function $(\Phi>\mathrm{h} \nu)$ in the middle of the spot (only 2PPE) and a lower work function moving away from the spots center (when $\Phi<\mathrm{h} \nu$ 1PPE is also possible). A simulated spectrum is shown in Fig. 2(b) together with the data. The 1PPE and 2PPE contributions in Fig. 2(d) show that 1PPE leads to the peak-like feature observed in the data, while the wider signal exhibiting a broadened low energy cut-off results from $2 \mathrm{PPE}$.

In the simulation, a constant density of states (DOS) multiplied by a Fermi-Dirac distribution function is assumed for the initial occupied states inside the $\mathrm{Cu}(111)$ substrate. As we only detect (scattered) secondary electrons from the $\mathrm{Cu}(111)$ substrate due to their short mean free path, we neglect the real (non-constant) DOS and just model the transmission of the photoexcited electrons through the rather thick $(>20 \mathrm{BL}) \mathrm{D}_{2} \mathrm{O}$ adlayer by multiplying with an exponential decay $\propto \mathrm{e}^{-E}$. This describes the typical shape of inelastically scattered secondary electrons in low energy photoemission spectroscopy well ${ }^{20}$. To get to the final energy scale, the resulting intensity is then shifted one (two) times the photon energy up in energy according to the order of the photoemission process. It is finally convolved with the energy bandwidth of the laser pulse and the low energy side of the spectrum is cut at the value of the work function to give the photoemission yield for $1 \mathrm{PPE}$ and $2 \mathrm{PPE}$. The ratio between 1PPE and 2PPE yield is a free parameter in our model and depends on various experimental conditions (e.g. laser fluence, cross sections, etc.). The total yield is obtained by numerical integration over the illuminated spot weighted with the illuminated area. Finally, the instrument function is included by convolving the result with the effective energy resolution of the hemispherical electron energy analyzer used in the experiment (in our case $80 \mathrm{meV}$ ). Our simple simulation describes the data remarkably well in the low energy region of the spectra shown in Fig. 2(b). The peak $\mathrm{e}_{\mathrm{T}}$ is not included in the model and resembles 
an unoccupied state in $\mathrm{D}_{2} \mathrm{O} / \mathrm{Cu}(111)^{21}$. Its discussion exceeds the scope of this paper.

To confirm the validity of our simulations, we test the model for different functional dependencies $\Phi(I)$ and parameters in Fig. 3 that define how the intensity profile of the laser (Fig. 3(a)) is translated into a work function distribution (Fig. 3(b)). First, we show the exponential dependence (proposed in Equation 1) in Fig. 3(c) and vary the exponential factor $\gamma$, resulting in increasingly curved/steep work function dependecies as depicted by the curves in the inset. In the case of a photoinduced increase of the work function (red and yellow curves) the threshold work function $(\Phi(I)=\mathrm{h} \nu)$ is reached already for lower normalized laser intensities the larger the $\gamma$. This is resulting in a steeper low-energy electron cut-off for the 2PPE part in the simulated spectrum and a more pronounced gap-like shape. For a photoinduced decrease (blue curves) there is no pronounced gap and $\gamma$ mainly tunes the intensity ratio between peak-shaped 1PPE and wider 2PPE part.

In addition, we show simulated spectra for a linear relationship between work function change and laser intensity $\left(\Phi(I)=\Phi_{0}+\Delta \Phi_{\max } \times I\right)$ in Fig. 3(d). Starting with the photoinduced increase (red curves), the gap-like shape is still reproduced, but the intensity in the crossover region between $1 \mathrm{PPE}$ and $2 \mathrm{PPE}$ differs from the one simulated on the basis of Equation 1. Lastly, if the sign of the work function change is inversed (photoinduced work function decrease - blue curves) a peak-like feature coming from the single-photon process remains, but the gap between the two orders is washed out, quite similar to the case of the exponentially decreasing $\Phi$ discussed above. Note that in the limit that $\gamma$ tends to zero, Equation 1 results in the linear dependence.

As mentioned before, the specific functional dependence is related to the mechanism of the work function change of the system under investigation. Nevertheless, for a system where $\Phi(I)$ is monotonically increasing, a peak and gap like shape is always observed.

We finally show simulation results for different maximum work function shifts $\Delta \Phi$ in Fig. $3(\mathrm{e}) . \Phi$ ranges from $4.5 \mathrm{eV}$ up to $5.5 \mathrm{eV}$ for the largest work function shift $\Delta \Phi$ (violet curve). The higher the value of $\Delta \Phi$ the bigger the 'gap size' between main $1 \mathrm{PPE}$ and $2 \mathrm{PPE}$ contributions. The maximum value of $\Phi$ (indicated by the dashed lines) for each of the simulated curves does not correspond to the energetic position of the low-energy cut-off at half-maximum intensity but to the top of the edge. This is counterintuitive and differs from the common definitions of edge positions (i.e. half-maximum or point of curvature-change).

In conclusion, we have shown that a spatially non-uniform work function strongly af- 
fects spectroscopic signatures in UPS and 2PPE spectroscopy. The most prominent effect is observed using a photon energy close to the varying work function of the system, due to a mixture of single- and two-photon processes. Based on a very simple model we describe the shape of the low-energy region of our photoemission data of $\mathrm{D}_{2} \mathrm{O} / \mathrm{Cu}(111)$ remarkably well without taking into account any features of the band structure. A quantitative analysis of such spectra can help to understand the underlying physics of the photoinduced work function change of a system. In the case of a photoinduced work function increase the general shape exhibiting two seperate low-energy cut-offs for 1PPE and (at a higher energy) for $2 \mathrm{PPE}$ intensity is observed. This is very robust against modifications of most model parameters (e.g. initial DOS, functional dependence of $\Phi$ ). We are therefore confident, that our model is able to explain a variety of effects observed in $2 \mathrm{PPE}$ experiments investigating photoreactive systems ${ }^{22}$.

We have furthermore demonstrated, that a varying work function may be easily overlooked in UPS measurements while strongly affecting the experimental results and interpretations. The shape and position of the low-energy cut-off of UPS spectra depends very much on the details of the work function distribution within the illuminated area. The light used for photoemission itself can induce a persistent modification of the work function and not only affect the resolution of the measurement but even change physical properties of the sample. We think that these effects should be considered when investigating energy level alignment in photoactive systems.

\section{REFERENCES}

${ }^{1}$ G. Heimel, I. Salzmann, S. Duhm, and N. Koch, Chem. Mater. 23, 359 (2011).

${ }^{2}$ N. Koch, A. Kahn, J. Ghijsen, J.-J. Pireaux, J. Schwartz, R. L. Johnson, and A. Elschner, Appl. Phys. Lett. 82, 70 (2003).

${ }^{3}$ B. Bröker, R.-P. Blum, J. Frisch, A. Vollmer, O. T. Hofmann, R. Rieger, K. Müllen, J. P. Rabe, E. Zojer, and N. Koch, Appl. Phys. Lett. 93, 243303 (2008).

${ }^{4}$ S. Schuppler, N. Fischer, T. Fauster, and W. Steinmann, Phys. Rev. B 46, 13539 (1992).

${ }^{5}$ W. Berthold, F. Rebentrost, P. Feulner, and U. Höfer, Appl. Phys. A 78, 131 (2004).

${ }^{6}$ J. Bardeen, Phys. Rev. 49, 653 (1936).

${ }^{7}$ N. D. Lang and W. Kohn, Phys. Rev. B 3, 1215 (1971). 
${ }^{8}$ R. Gerlach and T. Rhodin, Surf. Sci. 19, 403 (1970).

${ }^{9}$ T. C. Leung, C. L. Kao, W. S. Su, Y. J. Feng, and C. T. Chan, Phys. Rev. B 68, 195408 (2003).

${ }^{10}$ L.-M. Wang, V. Sametoglu, A. Winkelmann, J. Zhao, and H. Petek, J. Phys. Chem. A 115, 9479 (2011).

${ }^{11}$ H. Bluhm, T. Inoue, and M. Salmeron, Surf. Sci. 462, L599 (2000).

${ }^{12}$ I. H. Campbell, S. Rubin, T. A. Zawodzinski, J. D. Kress, R. L. Martin, D. L. Smith, N. N. Barashkov, and J. P. Ferraris, Phys. Rev. B 54, R14321 (1996).

${ }^{13}$ S. Duhm, G. Heimel, I. Salzmann, H. Glowatzki, R. L. Johnson, A. Vollmer, J. P. Rabe, and N. Koch, Nat. Mater. 7, 326 (2008).

${ }^{14}$ M. Westley, R. Baragiola, R. Johnson, and G. Baratta, Planet. Space Sci. 43, 1311 (1995).

${ }^{15}$ J. Stähler, M. Meyer, U. Bovensiepen, and M. Wolf, Chem. Sci. 2, 907 (2011).

${ }^{16}$ M. Wolf and P. Tegeder, Surf. Sci. 603, 1506 (2009).

${ }^{17}$ M. Bertin, M. Meyer, J. Stähler, C. Gahl, M. Wolf, and U. Bovensiepen, Faraday Discuss. 141, 293 (2009).

${ }^{18}$ J. Faure, J. Mauchain, E. Papalazarou, W. Yan, J. Pinon, M. Marsi, and L. Perfetti, Rev. Sci. Instrum. 83, 043109 (2012).

${ }^{19}$ T. Fauster, Prog. Surf. Sci. 46, 177 (1994).

${ }^{20}$ D. M. Alloway, M. Hofmann, D. L. Smith, N. E. Gruhn, A. L. Graham, R. Colorado, V. H. Wysocki, T. R. Lee, P. A. Lee, and N. R. Armstrong, J. Phys. Chem. B 107, 11690 (2003).

${ }^{21}$ The signal $\mathrm{e}_{\mathrm{T}}$ resembles electrons trapped at the $\mathrm{D}_{2} \mathrm{O} /$ vacuum interface similar to trapped electrons on $\mathrm{D}_{2} \mathrm{O}$ crystallites on $\mathrm{Ru}(001)^{23}$.

${ }^{22}$ M. P. Steele, M. L. Blumenfeld, and O. L. Monti, J. Phys. Chem. Lett. 1, 2011 (2010).

${ }^{23}$ U. Bovensiepen, C. Gahl, J. Stähler, M. Bockstedte, M. Meyer, F. Baletto, S. Scandolo, X.-Y. Zhu, A. Rubio, and M. Wolf, J. Phys. Chem. C 113, 979 (2009). 

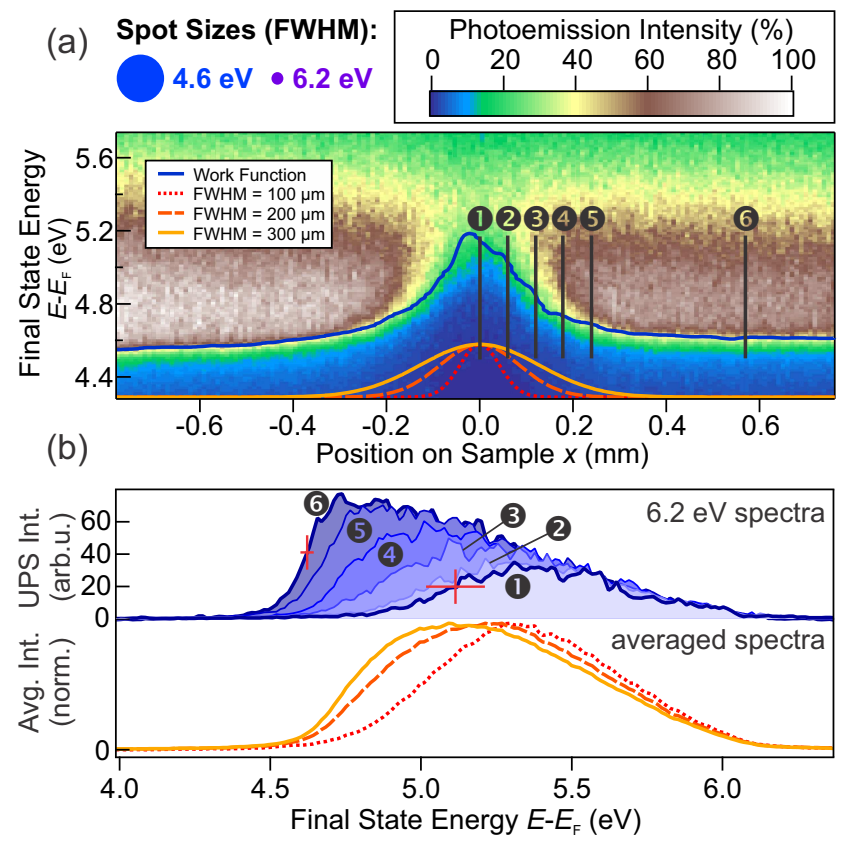

FIG. 1. (color online) (a) The false color plot shows the low energy region of 1PPE/UPS spectra of a $6.2 \mathrm{eV}$ beam scanning over a spot previously illuminated by a large $4.6 \mathrm{eV}$ beam (average power: $1.3 \mathrm{~mW}$ ) as a function of sample position $x$. The energetic position of the low-energy cutoff corresponds directly to the local work function (blue curve). (b) spectra at indicated locations (top) and averaged spectra over different probing spot sizes (bottom) are shown to illustrate the implications of work function changes induced on this system. 


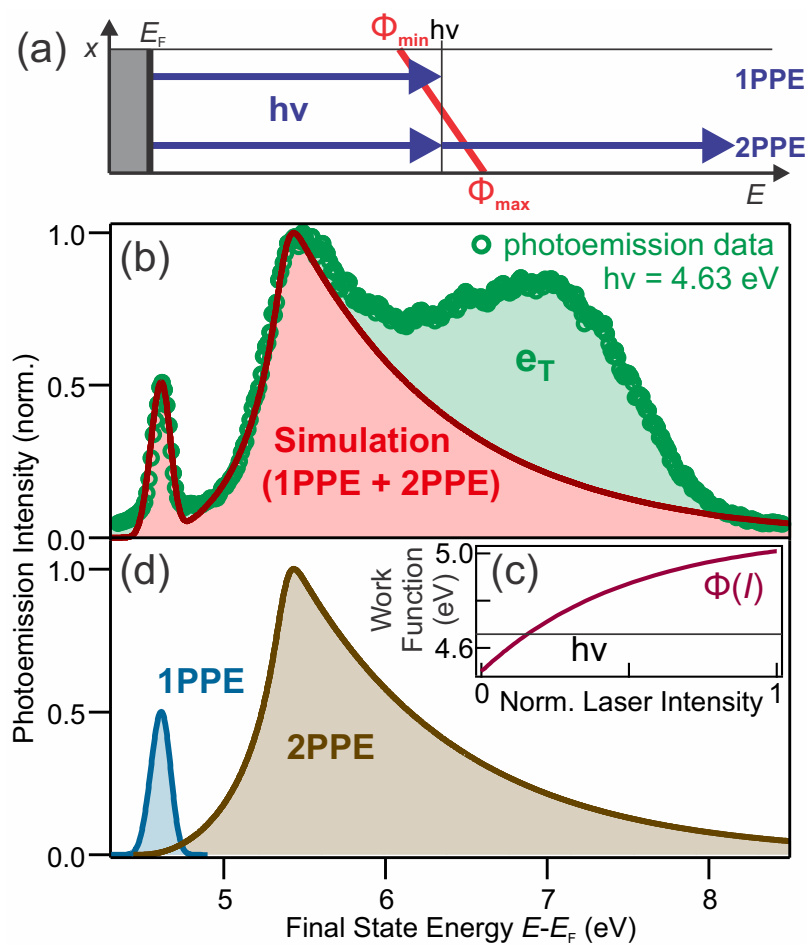

FIG. 2. (color online) (a) The work function $\Phi$ varies (between $\Phi_{\min }$ and $\Phi_{\max }$ ). 1PPE dominates the signal if $\Phi<\mathrm{h} \nu$, only 2PPE is possible if $\Phi>\mathrm{h} \nu$. (b) Photoemission data and simulation result. The trapped electron peak $\mathrm{e}_{\mathrm{T}}$ is not included in the model. (c) Intensity-dependent work function distribution. (d) Contributions of $1 \mathrm{PPE}$ and $2 \mathrm{PPE}$ to the model calculation. 

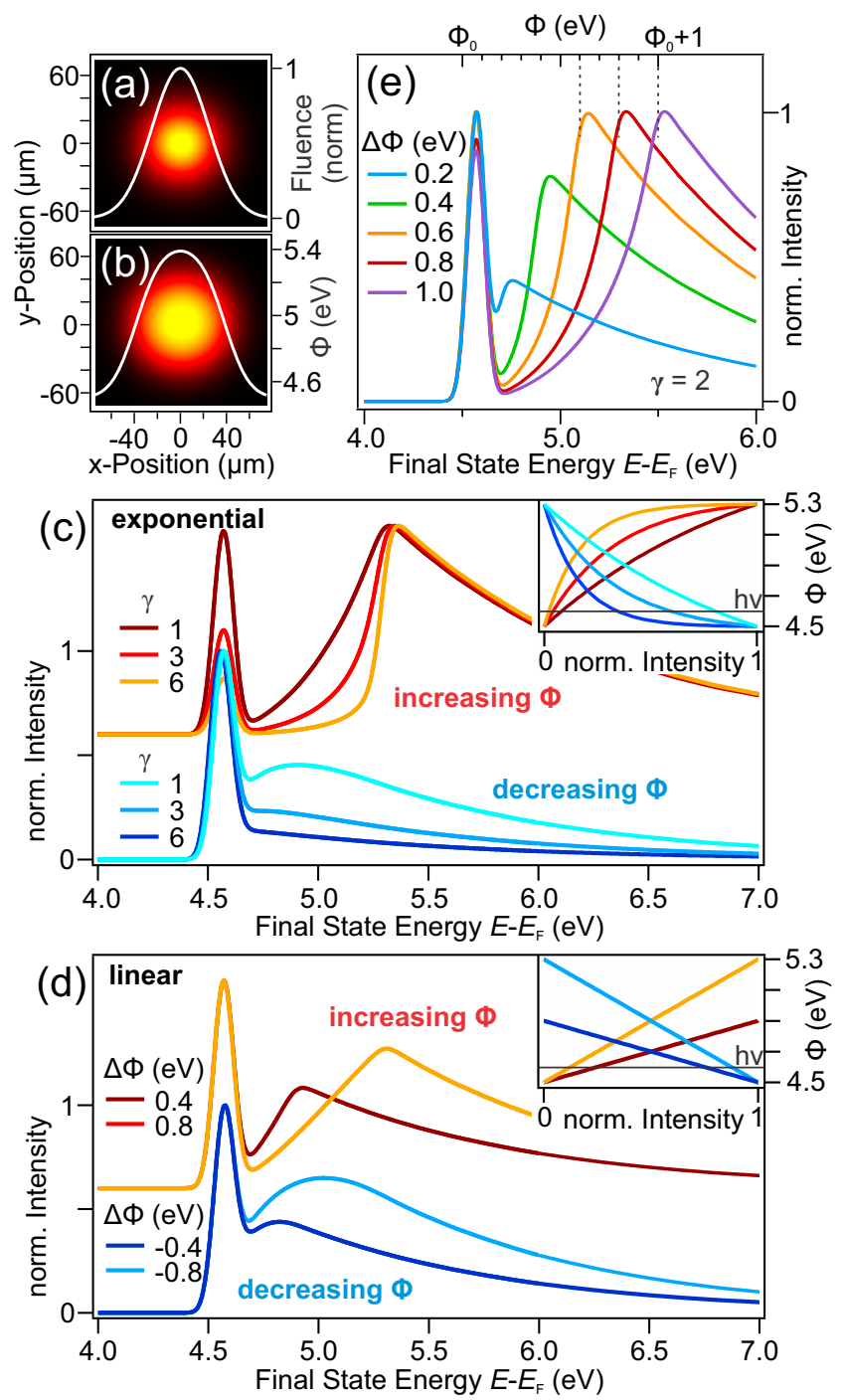

FIG. 3. (color online) (a) Gaussian laser intensity distribution, (b) resulting work function distribution. Simulated spectra based on exponential (c) and linear (d) functional dependencies (insets) of fluence and work function lead to similar qualitative shape of the low-energy region of the spectra. (e) Influence of $\Delta \Phi$ on the gap size. 